\title{
Bayesian Filtering of Surface EMG for Accurate Simultaneous and Proportional Prosthetic Control
}

\author{
David Hofmann, Ning Jiang, Senior Member, IEEE, Ivan Vujaklija, Student Member, IEEE, and \\ Dario Farina, Senior Member, IEEE
}

\begin{abstract}
The amplitude of the surface EMG (sEMG) is commonly estimated by rectification or other nonlinear transformations, followed by smoothing (low-pass linear filtering). Although computationally efficient, this approach leads to an estimation accuracy with a limited theoretical signal-to-noise ratio (SNR). Since sEMG amplitude is one of the most relevant features for myoelectric control, its estimate has become one of the limiting factors for the performance of myoelectric control applications, such as powered prostheses. In this study, we present a recursive nonlinear estimator of sEMG amplitude based on Bayesian filtering. Furthermore, we validate the advantage of the proposed Bayesian filter over the conventional linear filters through an online simultaneous and proportional control (SPC) task, performed by eight able-bodied subjects and three below-elbow limb deficient subjects. The results demonstrated that the proposed Bayesian filter provides significantly more accurate SPC, particularly for the patients, when compared with conventional linear filters. This result presents a major step toward accurate prosthetic control for advanced multi-function prostheses.
\end{abstract}

Index Terms-Bayesian filter, EMG amplitude estimation, simultaneous and proportional control.

\section{INTRODUCTION}

T HE surface myoelectric signal (sEMG) carries neural control information from the central nervous system and therefore can be used to infer and estimate motion intentions. For this reason, the sEMG has been used as a control signal for multi-function upper limb prostheses for more than six decades and is currently the only reliable control source for this application [1], [2]. Currently, almost all commercial prostheses still use a very basic sEMG processing scheme. With this approach, the control can only be sequential and for a limited number

Manuscript received March 31, 2015; revised September 29, 2015; accepted November 11, 2015. Date of publication November 20, 2015; date of current version December 06, 2016. This work was supported in part by the European Research Council (ERC) via the ERC Advanced Grant DEMOVE 267888, by the Federal Ministry of Education and Research (BMBF) of Germany under Grant 01GQ0811, and by the New Faculty Startup Grant of the University of Waterloo.

D. Hofmann was with the Department of Nonlinear Dynamics, Max Planck Institute for Dynamics and Self-Organization, Göttingen, Germany. He is now with the Department for Theoretical Physics, Emory University, Atlanta, GA 30322 USA (e-mail: david.hofmann@emory.edu).

N. Jiang is with Department of Systems Design Engineering, University of Waterloo, Waterloo, ON, N2L 3G1 Canada (e-mail: ning.jiang@uwaterloo.ca).

I. Vujaklija and D. Farina are with the Department of Neurorehabilitation Engineering, University Medical Center, Göttingen, Germany (e-mail: ivan.vujaklija@bccn.uni-goettingen.de; dario.farina@bccn.uni-goettingen.de).

Color versions of one or more of the figures in this paper are available online at $\mathrm{http}: / /$ ieeexplore.ieee.org.

Digital Object Identifier 10.1109/TNSRE.2015.2501979 (usually not greater than two) of degrees-of-freedom (DoF). The switch between DoFs is usually based on counter-intuitive muscle activations, such as co-activation of agonist/antagonist [3]. Due to the limited functionality and unnatural control, the patient compliance of these prostheses is low and $\sim 75 \%$ of upper limb amputees choose not to use active prostheses [4].

Because of the problems outlined previously, there has been an interest in the research community to improve the functionality and usability of multi-function prostheses, by better extracting neural control information with advanced signal processing and machine learning methods. After many decades of research and development, pattern recognition based methods show the first signs of potential clinical applications, with pilot systems being introduced into the market [5]. However, there are some inherent limitations of the pattern recognition-based methods. One of these limits is that these algorithms can only provide sequential control of multiple functions, while the neuromuscular system controls multiple physiological DoFs with a simultaneous and proportional (SPC) approach [1]. More recently, various approaches have been reported to realize SPC from the sEMG, either with regression approaches [6]-[8], or with modifications of the classic pattern recognition paradigm [9], [10].

Currently, all the approaches for EMG control are based on the extraction of features from the EMG signal and almost all of them use the signal amplitude or power estimation. Being a stochastic process, the amplitude of the sEMG is its standard deviation that can be estimated with estimators such as the mean absolute value (MAV) or root mean square value (RMS) [11]. Signal amplitude is a key feature in classic pattern recognition and essentially the only feature for regression-based methods [6], [12]-[14] in myocontrol. Although simple to calculate, classic estimators of EMG amplitude have high variability, which can be quantified by deriving the theoretical signal-to-noise ratio of the estimate [15]-[17]

$$
\mathrm{SNR}=\frac{E\left[\sum_{t-T}^{T} y^{2}(t)\right]}{\operatorname{Var}\left[\sum_{t-T}^{T} y^{2}(t)\right]}=B_{s} T
$$

where $y(t)$ is the sEMG signal, $B_{s}$ is its statistical equivalent bandwidth, and $T$ is the width of the processing window. To reduce the variability of the estimate, the effective bandwidth $B_{s}$ can be increased by whitening [18]. This strategy has been proven effective in pattern recognition-based myoelectric control algorithms [18]. Alternatively, varying $T$ will also influence the SNR of the estimate but this can be done within strict limits since a too long interval would increase the delay in the control 
output. This limitation is essentially the same as that associated to force/torque estimation from sEMG [19].

Alternatives of sEMG amplitude estimation to the traditional approach of RMS or MAV have been proposed, including a Bayesian-based estimator designed for detection of the EMG onset [20]. More recently, Bayesian filtering was specifically designed for sEMG amplitude estimation during dynamic contractions [21]. In the latter study, it was shown that the Bayesianbased amplitude estimator outperformed RMS and MAV, both in terms of stability and responsiveness [22]. However, the study in [22] was offline and was conducted only for one channel, one muscle, and with a simple ramping-up task.

In this study, we modified the Bayesian filtering approach proposed in [22] and applied it in an SPC framework. This is, to the best of our knowledge, the first time that Bayesian filtering was implemented and systematically evaluated in an online SPC experiment for myoelectric control.

\section{Methods}

\section{A. Bayesian Filtering of sEMG}

In the following, we present a formal framework of Bayesian filtering for surface EMG with some differences with respect to the presentation in [22]. Rather than a Fokker-Planck equation, we propose to use the differential Chapman-Kolmogorov equation [23] based on Markov processes, because formally the evolution equation presented in [22] is not a Fokker-Planck equation (which describes diffusion processes but not finite jump processes [24]) and is not derivable from the stochastic differential equation presented therein.

Given a recording of sEMG, denoted by $y(t)$, and the underlying "neural drive", denoted by $x(t)$, we have the following model:

$$
\begin{aligned}
& x(0) \rightarrow x(1) \rightarrow x(2) \rightarrow \cdots \rightarrow x(t) \rightarrow \cdots \\
& \downarrow \quad \downarrow \quad \downarrow \\
& y(1) \quad y(2) \quad y(t) \text {. }
\end{aligned}
$$

Let us assume that both are Markov processes such that

$$
\begin{aligned}
p(x(t+1) \mid x(t) \ldots x(0)) & =p(x(t+1) \mid x(t)) \\
p(y(t) x(t) \ldots x(0)) & =p(y(t) \mid x(t)) \\
p(x(t+1) \mid x(t), y(t) \ldots y(1)) & =p(x(t+1) \mid x(t))
\end{aligned}
$$

where $p(\cdot \mid \cdot)$ denotes the conditional probability density function. Our goal is to estimate $p(x(t) \mid \mathcal{D})$, where $\mathcal{D}(t)=$ $\{y(1), y(2), \ldots, y(t)\}$, i.e., the probability of the underlying neural drive taking a certain value, given the measured sEMG signal. We can express this conditional density by the densities given in (3) using the Bayes formula

$$
\begin{aligned}
p(x(t+1) \mid \mathcal{D}(t+1)) & \\
& =\frac{p(y(t+1) \mid x(t+1)) p(x(t+1) \mid \mathcal{D}(t))}{p(y(t+1) \mid \mathcal{D}(t))} .
\end{aligned}
$$

For recursive estimation of the amplitude $x$ with $p(x \mid \mathcal{D})$ at each point in time, we need to define two probability densities as can be seen from (3) and (4) (the denominator in (4) is just a normalization factor). We get the prior density of the current time step in (4) from the posterior density of the previous time step by applying a model for the temporal evolution, i.e., $p(x(t+$ 1) $\mid x(t))$. Additionally, we need to define a likelihood model in order to have all terms in the Bayes formula (4) set which allows us to update our estimates with the measurements. This two-step procedure is also termed evolution and observation model and defines the general procedure for Bayesian filters [25]. Besides the filter proposed in [22] and here, also the well-known Kalman filters and particle filters belong to this family of filters [25].

The temporal evolution is, as proposed in [22], assumed to be stochastic and governed by sudden jumps and diffusion. Such a stochastic process can be described by a differential ChapmanKolmogorov equation (the derivation based on Markov processes can be found in [23]). The general form of this equation is as follows:

$$
\begin{aligned}
\frac{\partial}{\partial t} p( & x(t+1) \mid x(t)) \\
= & -\sum_{i} \frac{\partial}{\partial x_{i}(t+1)}\left[A_{i}(x(t+1)) p(x(t+1) \mid x(t))\right] \\
& +\sum_{i, j} \frac{1}{2} \frac{\partial^{2}}{\partial x_{i}(t+1) \partial x_{j}(t+1)} \\
& \times\left[B_{i j}(x(t+1)) p(x(t+1) \mid x(t))\right] \\
& +\int_{0}^{x_{\max }} d z[W(x(t+1) \mid z) p(z \mid x(t)) \\
& -W(z \mid x(t+1)) p(x(t+1) \mid x(t))] .
\end{aligned}
$$

By assuming a 1-D 1 system and by setting the drift coefficient to $A_{i}(x(t+1))=0$, the diffusion coefficient to $B_{i j}(x(t+1))=$ $2 \alpha$, and the jump rates to $W(x(t+1) \mid z)=W(z \mid x(t+1))=$ $\beta / x_{\max }$, we recover the time evolution equation, also reported in [22]

$$
\begin{aligned}
\frac{\partial}{\partial t} p(x(t+1) \mid x(t))= & \alpha \frac{\partial^{2}}{\partial x^{2}(t+1)} p(x(t+1) \mid x(t)) \\
& +\beta\left(\frac{1}{x_{\max }}-p(x(t+1) \mid x(t))\right) .
\end{aligned}
$$

With respect to the equation derived in [22], however, the support of the stochastic variable is now set to $\left[0, x_{\text {max }}\right]$ instead of $[0,1]$ and, hence, an additional $1 / x_{\max }$ term appears in (6) which assures conservation of the probability under the evolution equation.

The likelihood $p(y(t) \mid x(t))$ can take several forms, such as [22], is shown as follows:

$$
\begin{aligned}
& p(y(t) \mid x(t))=\frac{x(t)^{n} e^{-x(t)}}{n !} \\
& p(y(t) \mid x(t))=\frac{e^{\frac{-y^{2}(t)}{2 x^{2}(t)}}}{\sqrt{2 \pi x^{2}(t)}} \\
& p(y(t) \mid x(t))=\frac{e^{\frac{(y(t)}{x(t)}}}{x(t)} .
\end{aligned}
$$

Equations (7), (8) and (9) are referred to as the Poisson model, Gaussian model, and double exponential or Laplacian model, respectively, which are well grounded from previous sEMG research [26]. By discretizing in space and time (6) and deciding 
on a point estimation method based on the posterior distribution evaluated at each time step, we get the following recursive algorithm:

1) Time evolution updating:

$$
\begin{aligned}
p(x(t+1) \mid \mathcal{D}(t)) & \\
=(\alpha & \frac{p(x(t)+\Delta x(t)}{\Delta x^{2}(t)} \\
& +\alpha \frac{p(x(t)-\Delta x(t))-2 p(x(t))}{\Delta x^{2}(t)} \\
& \left.+\beta\left(\frac{1}{x_{\max }}-p(x(t))\right)\right)+p(x(t))
\end{aligned}
$$

2) Observation updating:

$p(x(t+1) \mid \mathcal{D}(t+1))=\frac{p(y(t+1) \mid x(t+1)) p(x(t+1) \mid \mathcal{D}(t))}{p(y(t+1) \mid \mathcal{D}(t))}$

3) Point estimation:

$$
\hat{x}(t+1)=\sum_{x} x(t+1) p(x(t+1) \mid \mathcal{D}(t+1)) .
$$

In the current study, we chose the point estimation (see point 3 of the previous algorithm) to be the expectation value rather than the maximum of the posterior distribution that was proposed in [22]. This was necessary to reduce the frequent large sudden "jumps" in estimates produced by the latter choice during fast dynamic contractions. For example, Fig. 1 shows the estimated sEMG amplitude of four contractions. The first three contractions reached stable force levels and the last was a linearly increasing force contraction. A three-fold increase in the resolution (number of bins) of the amplitude axis $x$ smoothened small amplitude estimate variations but did not affect the large ones. Using the expectation value as point estimation, as proposed in this study, reduced the number and magnitude of the large sudden variations. The exponential distribution [see (9)] was chosen for a model of the likelihood as it resulted in better SNR. A uniform prior with resolution of 100 bins $\mathrm{p}(\mathrm{x}(0))=$ $x_{\max } / 100$ was set. From an offline analysis performed on pilot data, the values of the two parameters $\alpha$ and $\beta$ were set to $10^{-10}$ and $10^{-40}$, respectively. The offline analysis considered maximizing the SNR while minimizing the large "jumps" in estimates (see Fig. 1).

\section{B. Subjects}

Eight able-bodied volunteers (5M, 4F, 22-40 years old) and three subjects with limb deficiency participated in the experiments. Two of the limb-deficient subjects are transradial amputees, and one has congenital deformation of the right hand/ wrist. None of the participants had any known neurological disorders. At the time of participation, most of the able-bodied subjects had never participated in a myoelectric control experiment, while all amputees were users of commercial myoelectric prostheses, which operate in the classic one channel/one function regime with co-activation triggered mode switch. The subject with congenital deformation never used a prosthesis. Prior to the participation, all subjects read and signed the study protocol
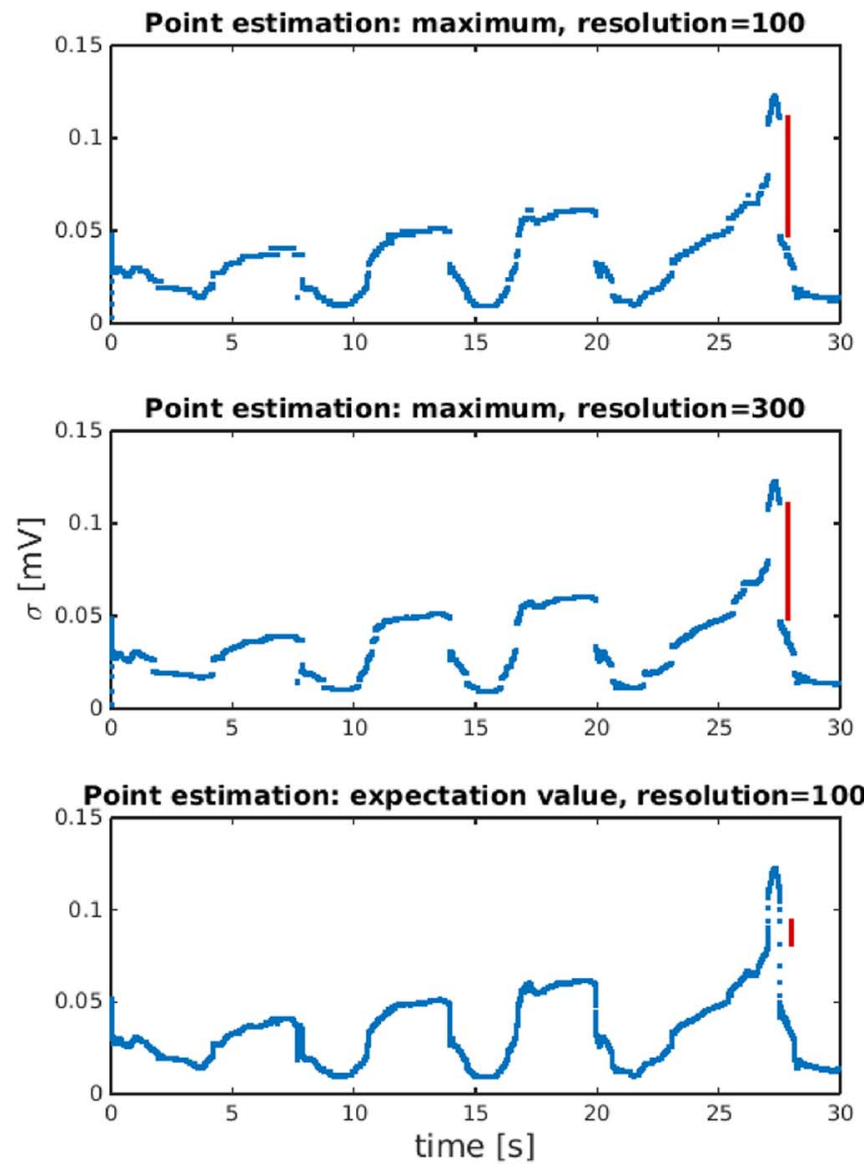

Fig. 1. Comparison of estimates with respect to fast variations of the amplitude. The same EMG signal was filtered using the maximum of the posterior distribution (as in Error! Reference source not found.) with a resolution of 100 bins (top). The resolution was then increased three-fold (middle panel) with the same approach. Finally, the expectation value was used for the point estimation, as suggested in this study, with 100 bins (bottom panel). The red bar marks the largest variation in the amplitude estimate trajectory.

and the informed consent form, which was approved by the research ethic committee of the University Medical Center Göttingen (No. 22/2/12).

\section{Experiment Procedures}

The experiment protocol was similar to those of previous studies on myoelectric SPC [7], [27], [28]. For the sake of clarity, the experimental procedure is again described here.

1) Instrumentation Setup: During the experiment, the subject was seated, with the dominant arm in the neutral position: arm at the side of the body, with the palm facing inward. Sixteen monopolar surface electrodes (Neuroline 720, Ambu, Denmark) were placed equidistantly in two rows, with eight electrodes per row. The two rows were centered approximately $1 / 3$ distal of the forearm, measured from the olecranon process to the styloid process of the ulnar bone. The inter-row distance was approximately $10 \mathrm{~mm}$, and the inter-electrode distance within each row varied from 25 to $35 \mathrm{~mm}$, depending on the circumference of the subject's forearm. The EMG signals were acquired by a bio-signal amplifier (EMGUSB2, OT Bioelettronica, Italy) at a sampling rate of $2048 \mathrm{~Hz}(12 \mathrm{bit} \mathrm{A} / \mathrm{D}, 3 \mathrm{~Hz}$ to $900 \mathrm{~Hz}$ sixth-order Butterworth band-pass, 500-1000 gain). During the 
experiment, instructions and virtual objects were displayed on a monitor, which was placed in front of the subject at a distance of 1-1.5 m. Raw EMG signals were displayed on a separate monitor to the experimenter, so the quality of the acquired signal was monitored constantly during the experiment. Each experimental session consisted of three phases: familiarization, calibration/training, and online control. Each phase of the experimental session contained one or more runs, i.e., a continuous data recording. The subject could request rests during a run at any time. During the session, the subject was instructed to perform a series of movements, activating either separately or simultaneously 2 DoFs of the dominant wrist.

2) Familiarization Phase: The first phase was the familiarization phase, during which the subject was introduced to the virtual environment, where a virtual arrow on the monitor could move left/right or rotate in clockwise and counter-clockwise direction. The subject was instructed that the left/right movement of the arrow would be associated to the wrist flexion and extension $(\mathrm{DoF} 1)$ in latter phases of the experiment, whereas the rotational movement would be associated to the wrist supination and pronation (DoF2). The displacement of the arrow from its original position would correlate to the effort of the contraction.

3) Calibration Phase: After the familiarization phase, the calibration phase would start. In this phase, the subject was cued to perform movements of DoF1 and DoF2, with the moving arrow displayed on the screen, i.e., the subject would follow the arrow, by performing corresponding single DoF wrist contractions. Each DoF movement was repeated for eight trials, and each trial lasted approximately $10 \mathrm{~s}$, with breaks in between at the request of the subject. The calibration phase consisted in the 16 trials and usually lasted less than $3 \mathrm{~min}$. The data recorded during this phase were used to calibrate the step-wise non-negative matrix factorization (NMF) regressor, which has been described previously [6], [7]. In short, it is a minimally supervised algorithm that is capable of estimating simultaneous activations of multiple DoFs by using multi-channel EMG recorded during single DoF contractions. In the current study, two features were used to calibrate the NMF regressor: the classic MAV feature used in earlier studies and the estimates obtained using the proposed Bayesian filter. Both features were calculated at the same rate (with $140 \mathrm{~ms}$ window, $100 \mathrm{~ms}$ overlapping, equivalent to 25 $\mathrm{Hz}$ ). Therefore, two estimators were obtained for each subject, which are referred to as MAVe and BAYe hereinafter. Once the two regressors were calibrated, the online control phase would start immediately.

4) Online Control Phase: During this phase, with the regressors calibrated, the EMG data generated by the subject were translated into a 2-D control signal that represented the estimated activation intentions of the subject in the two DoFs in real time. These estimates were mapped to the movements of the virtual arrow, i.e., the subject controlled the movements of the arrow, rather than following the movement of the arrow as in the calibration phase (see Fig. 2). Now, in addition to the arrow, in the online control phase, a series of circular targets were used. The subject was instructed to hit the targets with the tip of the arrow and to remain within their reach for at least $300 \mathrm{~ms}$ (the dwelling time). If this task was accomplished in less than 20 $\mathrm{s}$, the target hitting was considered successful, as also described

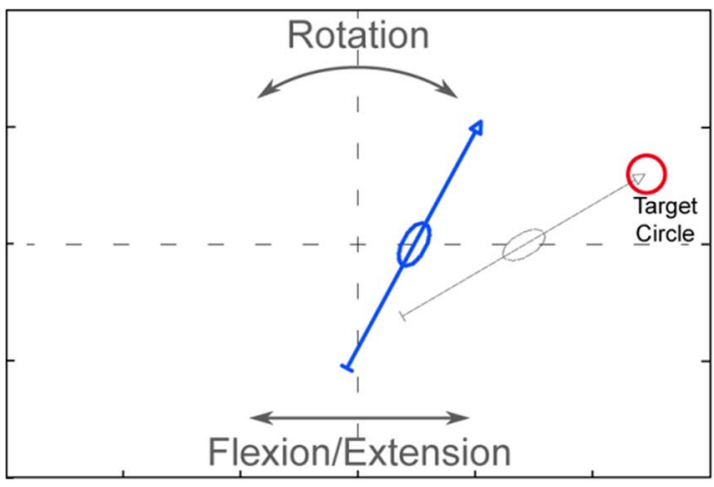

Fig. 2. Feedback presented to the subject during the experiment. Left-right movements of the arrow represented wrist flexion and extension (DoF1), and the rotational movements of the arrow represented wrist rotation (DoF2). A representative large target of type T3, which required the activation of both DoFs, is illustrated as well.

in previous studies [6], [27], [28]. When the 20-s period elapsed without success, the trial was considered as failed. In the present study, we further implemented two target sizes, which differed from the previous studies. The large target had a diameter of 8 density-independent pixels (dp) [29], and the small target was 4 $\mathrm{dp}$ in diameter. As the entire working space for the subject, i.e., the space where the tip of the arrow could reach, was $360 \times 60$ $\mathrm{dp}$, the areas of the targets were only $1 \%$ and $0.25 \%$ of the entire working space, for the large and small targets, respectively. For each size, three target types were presented to the subject. To reach the first type of targets (T1), the subject only needed to activate DoF2 (wrist rotation), although he could decide to activate both DoFs. To reach the second type of targets (T2), the subject only needed to activate DoF1 (wrist flexion and extension), although he could decide to activate both DoFs. The third type of targets (T3) was placed in a way that the subject had to activate both DoFs simultaneously at least for a period during the trial. Twenty targets for each type and each size were presented to each subject, for MAVe and BAYe. The set of targets was identical for the two regressors and for all subjects but were presented randomly in fixed subsets of four during the experiment for a specific algorithm and subject, so that it appeared to be random for the subject during the experiment, while it was still possible to do repeated measures in statistical analysis (see the following). The subject was blind as to which regressor was used during the experiment. The order of MAVe and BAYe for each subject was fully randomized. In this phase, the trajectory of the arrow tip, as well as the time of each trial, were recorded for further analysis, as detailed in the following. Different from our earlier studies [7], [27], [28], after each attempt, the subject did not have to return the arrow to the original position before attempting the next target but could just continue from one target to the next until the set of four would be finished. This strategy was chosen as it was more engaging for the subjects and it better simulated what could be translated into object manipulation task.

\section{Performance Analysis}

The trajectories of the arrow tip as well as the time for each trial in the online control phase were further processed offline, 
TABLE I

DEFINITIONS OF ONLINE PERFORMANCE INDICES

\begin{tabular}{|l|l|}
\hline $\begin{array}{l}\text { Completion rate } \\
(\alpha,[\%])\end{array}$ & $\begin{array}{l}\text { the number of completed tasks over the total } \\
\text { number of attempted tasks }\end{array}$ \\
\hline $\begin{array}{l}\text { Completion time } \\
\left(t_{c},[\mathrm{~s}]\right)\end{array}$ & $\begin{array}{l}\text { the time it took the subject to complete the } \\
\text { successful attempt }\end{array}$ \\
\hline Overshoots $(k)$ & $\begin{array}{l}\text { the number of occurrences that the tip of the } \\
\text { arrow passed through the target before the } \\
\text { dwelling time was reached }\end{array}$ \\
\hline $\begin{array}{l}\text { Throughput } \\
(T P,[\text { bit/s] })\end{array}$ & $\begin{array}{l}\text { the ratio of the task difficulty index }(T D) \text { of each } \\
\text { target and the completion time }\left(t_{c}\right)\end{array}$ \\
\hline $\begin{array}{l}\text { Efficiency } \\
\text { coefficient } \\
(\Gamma,[\%])\end{array}$ & $\begin{array}{l}\text { the ratio between the length of the optimal path } \\
\text { from the initial point to the target and the actual } \\
\text { trajectory realized (a value of } 100 \% \text { indicates a } \\
\text { perfect execution) }\end{array}$ \\
\hline
\end{tabular}

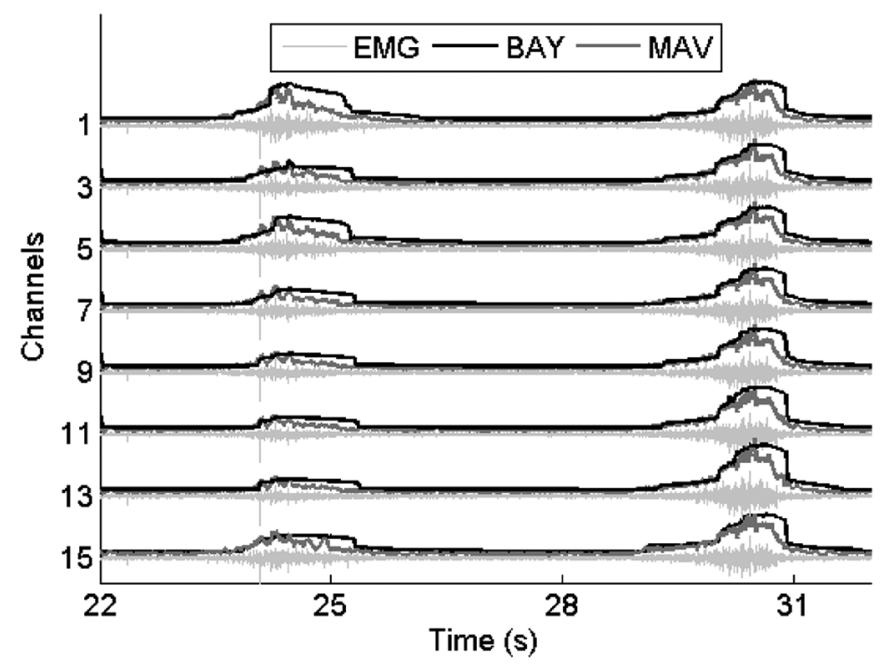

Fig. 3. Representative example of sEMG signal and the corresponding estimations with the proposed Bayesian filter and MAV, respectively. Data is from an amputee subject. Only 8 out of 16 channels are shown. Estimation by the Bayesian filter is smoother than MAV.

in order to analyze the control performance of each regressor under different conditions (target size and type).

Five online performance indices were used: completion rate $(\alpha)$, completion time $\left(t_{c}\right)$, overshoots $(k)$, throughput $(T P)$, and efficiency coefficient $(\Gamma)$, which are defined in Table I. It is important to note that $T P$ and $\Gamma$ in here is different from those defined in [30], as the coordinate system of the current study was not Cartesian, as in [30].

\section{E. Statistical Analysis}

The objective of the current study was to investigate if the two regressors, MAVe and BAYe, performed differently under different conditions (target size and type) during the online control experiment. For this purpose, three-way repeated measure ANOVA tests were conducted. Separate tests were performed for able-bodied and limb-deficient subjects. The response variables for the tests were the performance indices: $t_{c}, \Gamma, T P$ and $k$. The three factors of the ANOVA were target type (T1, T2 and T3), target size ( $\mathrm{S}$ and L, standing for small and large targets, respectively), and features (MAV and BAY). The repeated measure was done over subjects and targets, by setting these two factors as random factors. In all ANOVA tests, the full model was used first. When significant interaction(s) were detected, focused ANOVA was conducted by fixing the levels of one of the interacting factors. When no interaction was detected, the interaction terms were pooled, and the reduced ANOVA model with only the main factor was performed. Tukey comparison with Bonferroni correction was performed when a factor was found to be significant. The significance level for all tests was 0.05. All statistical analyses were performed using Minitab 16.

\section{Results}

\section{A. Descriptive Results}

Representative EMG signals and the estimated amplitudes using both MAV and Bayesian filter are shown in Fig. 3, using part of the calibration data from one amputee subject. The control signal obtained by the Bayesian filter was smoother than that of MAV, but also more responsive to fast changes. This observation is confirmed by the online trajectories reported in Fig. 4. The trajectories of BAYe are clearly smoother than those of MAVe and the superior responsiveness of BAYe is evident by the fact that the subject can start and stop the arrow with less overshoots. The difference between the two controllers is more pronounced when the fine control, i.e., small targets, is required (top-right and bottom-right panel of Fig. 4 for BAYe and MAVe, respectively). The five performance indices, $\alpha, t_{c}, \Gamma, T P$ and $k$, are summarized in Table II and Fig. 5, respectively.

\section{B. Completion Rate}

Completion rates for both subject groups, all target types and sizes can be found in Table I. In the able-bodied group, BAYe shows improvement over MAVe for large target size only in T3. However, when the target size is small a consistent improvement can be seen across all tasks. For limb-deficient subjects improvement is observed only for $\mathrm{T} 1 \mathrm{regardless}$ of size. This is probably due to the small sample size in this group.

\section{Completion Time}

For able-bodied subjects, there was no significant two-way or three-way interaction in the full model ANOVA for $t_{c}(p>$ $0.1)$. After pooling the interaction terms, the $t_{c}$ of BAYe was found to be significantly lower than for MAVe $(p<0.001)$. For limb-deficient subjects, target type was found interacting with the algorithm in $t_{c}(p=0.041)$, with no further interactions. Focused ANOVA on target type found that BAYe outperformed MAVe for T1 (DoF2 only) and T3 (combined DoF), with $p=0.001$ and $p=0.016$, respectively. For T2 (DoF1 only), the algorithm was not a significant factor $(p=0.492)$. Focused ANOVA on target size found that in both large and small target sizes, the algorithm had a significant effect on $t_{c}$, with $p=0.027$ and $p=0.005$, respectively. In summary, when using BAYe, both able-bodied and limb-deficient subjects could accomplish the tasks with a significantly shorter time than when using MAVe, in most cases.

\section{Path Efficiency}

For able-bodied subjects, no interaction was found in the fullmodel ANOVA. After pooling the interaction terms, the algorithm was found to be significant $(p<0.001)$, and BAYe out- 

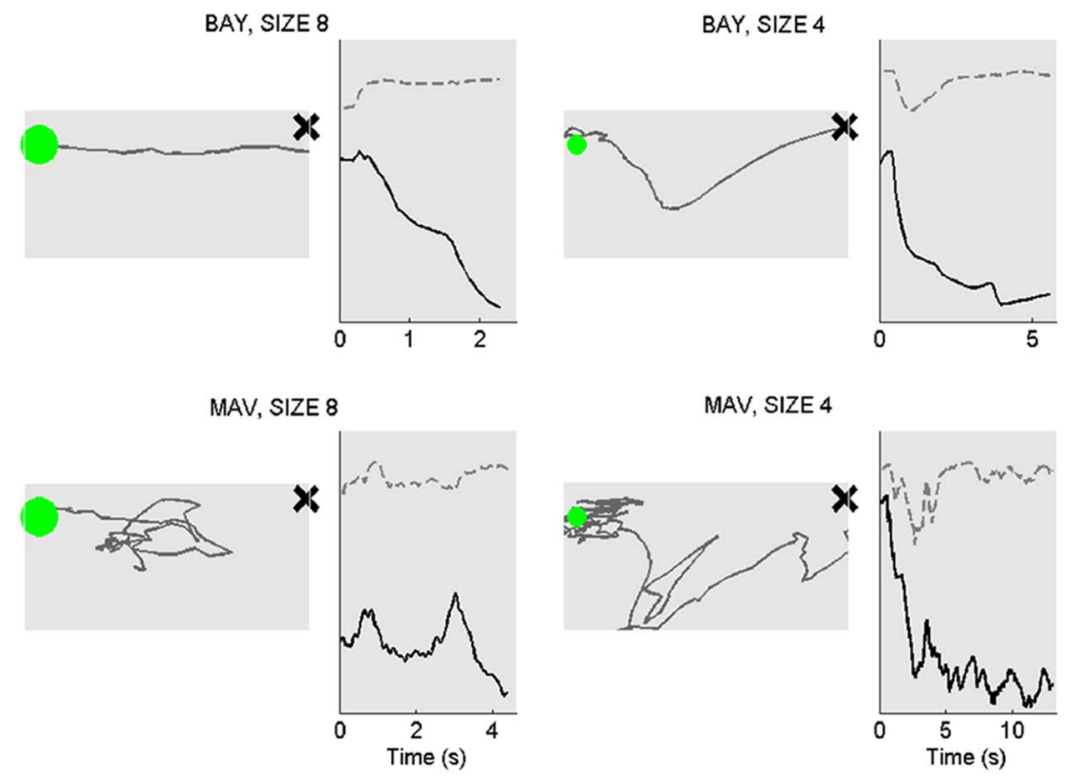

Fig. 4. Representative example of the trajectories of one of the amputee subjects, for a T3 target, using the two algorithms (top: Bayes; bottom: MAV), and when the target had two different sizes (left: large; right: small). In each scenario, the left plot is the trajectory in the 2-D working space. The cross indicates the resting position of the arrow, and the green dot indicates the position of the target circle. The plot on the right-hand side of each scenario is the time course plot of the $\mathrm{x}$ (solid line) and y (dashed line) position of the tip of the arrow. As described in the text, the subject started the task without necessarily returning the arrow to the rest position (e.g., in MAV size 8).

TABLE II

Completion Rates in Percentiles for Both Groups of Subjects, With Respect to All Target Types and Both Large (L) and Small (S) TARGET SIZES

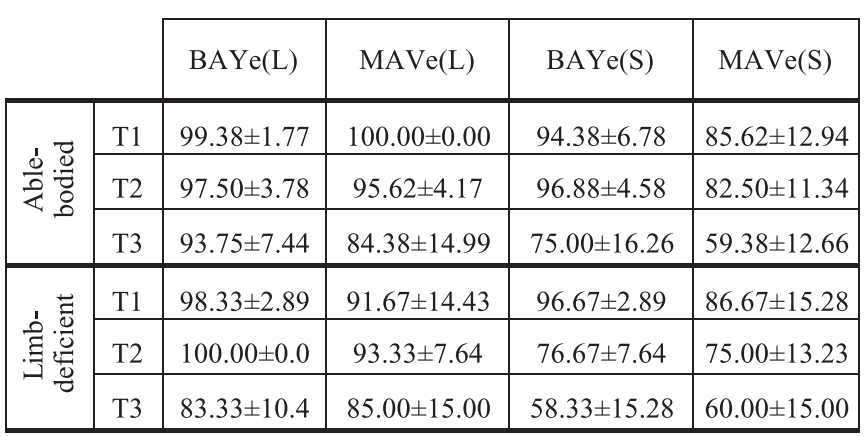

performed MAVe (the mean values of $\Gamma$ were 0.5 versus 0.4 , respectively). Similarly, for limb-deficient subjects, BAYe also outperformed MAVe $(p<0.001)$ in the path efficiency measure ( 0.4 versus 0.3$)$. In summary, BAYe provided significantly better path efficiency than MAVe, regardless of target type or target size, for both able-bodied subjects and limb-deficient subjects.

\section{E. Throughput}

For able-bodied subjects, the full model ANOVA found a significant interaction between target type and target size $(p=$ 0.003). Subsequent focused ANOVA found that only for target $\mathrm{T} 3$, the algorithm had a significant influence $(p=0.005)$. However, for limb-deficient subjects, the algorithm was found to be significant in the three-way analysis $(p=0.002)$, with no significant interaction $(p>0.1)$. The results indicated that BAYe's advantage over MAVe on Throughput is most apparent for limb-deficient subjects. For able-bodied subjects, BAYe outperformed MAVe when the task required simultaneous activations of both DoFs.

\section{F. Overshoot}

For able-bodied subjects, the algorithm interacted with both target type and target size in the three-way ANOVA, and with the remaining factor in all the subsequent focused two-way ANOVA. In the further focused one-way ANOVA, BAYe was consistently shown to have a smaller $k$ than MAVe $(p<0.001$ for all cases). For limb-deficient subjects, the focused ANOVA showed that BAYe had a smaller number of overshoots than MAVe for both target sizes $(p<0.001)$, and the same for all three target types $(p<0.001)$. Although the statistical analysis for overshoot seemed to be most complicated and less straightforward, it is clear, as shown in Fig. 5, that the values of $k$ in BAYe were remarkably smaller than those of MAVe. The interaction mainly stemmed from the fact that the extent of the difference of two features on $k$ depended on target type and target size. Clearly, the more difficult the tasks were, the greater advantage BAYe had over MAVe.

\section{DISCUSSION}

In this study, we present a novel approach using sEMG amplitude estimation within the framework of Bayesian filtering. The effectiveness of this approach in myoelectric control with SPC was systematically analyzed through goal-oriented online experiments, with both able-bodied subjects and below elbow limb-deficient subjects. We demonstrated that the proposed Bayesian filtering framework provides significant advantages over conventional amplitude estimators. Not only does it provide smoother estimates of the underlying "neural drive" from the sEMG but it is also more responsive to sudden changes in 

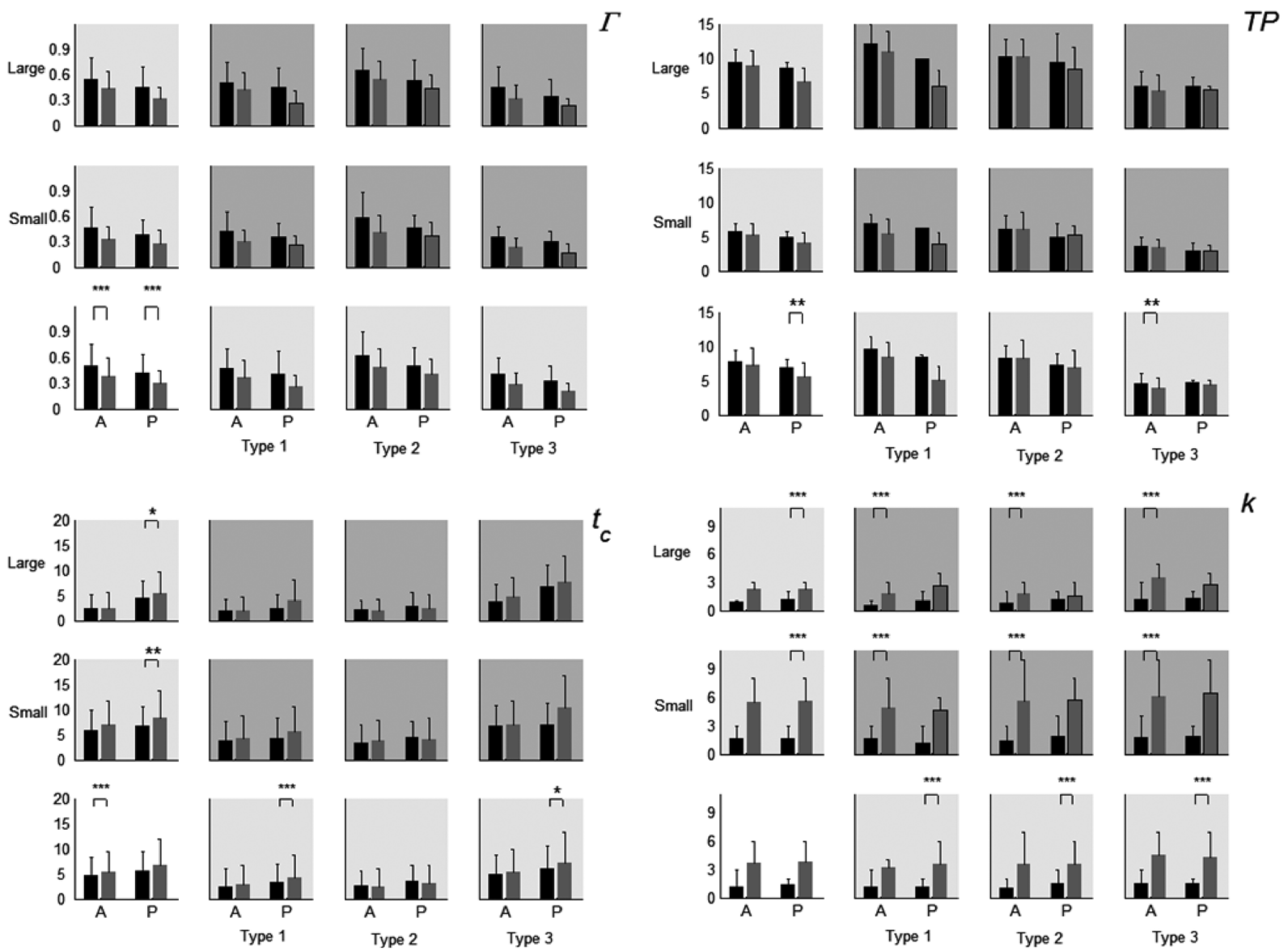

Fig. 5. Summary of the online performance results. The four panels are for $\Gamma, T P, t_{c}$, and $k$. Each panel is organized in a factorial design. The lower left plot is the grand average of the corresponding performance measure of the two algorithms: BAYe (black) and MAVe (gray). The top and middle rows are for large and small target, respectively, and the two left-most plots of these two rows are the average values for each size. The second, third and fourth columns from the left are for each target type, and the three plots in the bottom row are the average values for each type. The white background (the lower left) indicate the grand average; the light gray background indicates average of the case when the level of one of the factors is fixed, and the dark gray color indicates the average of the case when the levels of two factors are fixed. Stars denote the significance of ANOVA tests (*: $0.05>p>0.01$; $\left.^{* *} 0.01>p>0.001 ;{ }^{* * *}: p>0.001\right)$. "A" denotes able bodied subjects and "P" denotes limb-deficient subjects.

the neural drive. Consequently, when the proposed approach was used for SPC tasks, the subject was able to accomplish the tasks more efficiently. Our results also showed that the benefit of the proposed approach was more evident in subjects with limb deficiencies than able-bodied subjects.

\section{A. Bayesian Filtering and Conventional Linear Filters}

The conventional sEMG amplitude estimation follows the procedure of rectification (e.g., absolute value or square root) or other nonlinear transformations, followed by smoothing (lowpass filtering). As discussed in Section I, this approach suffers from the limited SNR [see (1)]. To improve the SNR, one could use a filter with a longer time constant, at the expense of less responsiveness. Prewhitening of the signal can improve it by increasing the statistical equivalent bandwidth of the signal. But this approach is still subject to the limitation of the low-pass filter, i.e., the compromise between the smoothness versus responsiveness. The proposed Bayesian filter allows control of these two factors separately, thanks to the two parameters in (6), i.e., $\alpha$ and $\beta$, respectively. The diffusion constant, $\alpha$, determines the smoothness of the estimate when the underlying neural drive is constant, while $\beta$ controls the possibility of sudden "jumps" and consequently the responsiveness of the estimator. Therefore, the proposed approach has the highly desirable property of achieving both smoothness and responsiveness simultaneously.

One potential advantage of the conventional approach is its low computational load. However, we have shown that a Matlab implementation of the proposed algorithm can run in real time on a regular PC (a $2.5 \mathrm{GHz}$ CPU was used for the experiments performed for this study). An optimized and compiled version of the algorithm would allow an embedded system implementation whose feasibility was shown already in [31]. Scripts of the Bayes Chapman Kolmogorov filter for EMG amplitude estimation can be found in the following repository: https://github. com/MPIDS/EMG-BayesFilter.git.

\section{B. Effectiveness in Myoelectric SPC}

Due to the property of achieving smoothness and responsiveness in the amplitude estimation, the proposed approach demonstrated a clear advantage over the conventional linear filter, such as MAV in the online myoelectric SPC experiment. In all online performance indices, BAYe showed statistically significant performance gain over MAVe. The subjects were able to perform the tasks faster (shorter task completion time), the trajectories were more economic (higher path efficiency), and it was easier 
to precisely place the arrow tip inside the target (less number of overshoots), with consequent overall higher control throughput. The advantages of BAYe were more evident when the task was complex, i.e., when the task required simultaneous activations of the both DoFs, and when the target was smaller. Further, the limb-deficient subjects seemed to benefit more from BAYe than able-bodied subjects, since in almost all cases BAYe showed statistically significant advantages over MAVe.

\section{Difference With Other Bayesian Filtering for EMG}

A few early works, using a similar approach, include Kalman filters for prosthetic control [20], [32], EMG prediction from cortical recordings [33], EMG change point estimation [21], Bayesian-based sensor fusion [34], [35], and recursive EMG amplitude estimation [22] on which our study is based. Among these, the recent work by Anugolu et al. [35] and Kumar et al. [34] were focused on offline analysis and therefore are difficult to compare against. Other studies applying [22] used it for proportional control of four finger functions [36], EMG driven biomechanical modelling of walking patterns [37] and biofeedback-based rehabilitation procedures [31], [38]. It should be noted that the time evolution model in [22] is, by definition, not a Fokker Planck equation, which only describes diffusion processes [24]. Instead, we proposed to use the differential Chapman-Kolmogorov equation, so as to provide a mathematically rigorous framework. Moreover, we propose a different point estimation method. We find that using the expectation value makes a significant difference as compared to the maximum of the posterior distribution proposed in [22]. It alleviates the detrimental effect of fast signal variations during control tasks that occur when the force level is changed fast. Indeed, we observed increased smoothness of the amplitude estimate with respect to sudden bursts of EMG signals using the proposed point estimation. Other studies achieved similar degrees of smoothness by clipping the raw EMG signal prior to any filtering [22], [36]. However, this ad hoc clipping approach cannot be used to track sudden decreases in the neural drive, contrary to the proposed algorithm (see Fig. 1). It might also obscure intended bursting neural commands, which can be useful in dexterous control.

\section{Conclusion}

In conclusion, a method for sEMG amplitude estimation based on Bayesian filtering and its application to SPC for active prostheses has been proposed and validated. It represents a step forward in the use of EMG for myocontrol by reducing its basic source of variability.

\section{ACKNOWLEDGMENT}

All authors declare no conflict of interests.

\section{REFERENCES}

[1] N. Jiang, S. Dosen, and D. Farina, "Myoelectric control of artificial limbs: Is there the need for a change of focus?," IEEE Signal Process. Mag., no. 5, pp. 12-15, Sep. 2012.

[2] E. Scheme and K. Englehart, "Electromyogram pattern recognition for control of powered upper-limb prostheses: State of the art and challenges for clinical use," J. Rehabil. Res. Dev., vol. 48, no. 6, p. 643, 2011.
[3] M. Asghari Oskoei and H. Hu, "Myoelectric control systems-A survey," Biomed. Signal Process. Contr., vol. 2, no. 4, pp. 275-294, 2007.

[4] D. Atkins, D. Heard, and W. Donovan, "Epidemiologic overview of individuals with upper-limb loss and their reported research priorities," JPO J. Prosthetics Orthot., vol. 8, no. 1, 1996.

[5] Coapt LLC 2015 [Online]. Available: http://www.coaptengineering com/

[6] N. Jiang, K. B. Englehart, and P. A. Parker, "Extracting simultaneous and proportional neural control information for multiple-DOF prostheses from the surface electromyographic signal," IEEE Trans. Biomed. Eng., vol. 56, no. 4, pp. 1070-1080, Apr. 2009.

[7] N. Jiang, H. Rehbaum, I. Vujaklija, B. Graimann, and D. Farina, "Intuitive, online, simultaneous, proportional myoelectric control over two degrees-of-freedom in upper limb amputees.," IEEE Trans Neural Syst. Rehabil. Eng., vol. 22, no. 3, pp. 501-510, May 2014.

[8] C. Castellini and P. van der Smagt, "Evidence of muscle synergies during human grasping," Biol. Cybern., vol. 107, no. 2, pp. 233-245, 2013.

[9] A. Young, L. Smith, E. Rouse, and L. Hargrove, "Classification of simultaneous movements using surface EMG pattern recognition," IEEE Trans. Biomed. Eng., vol. 60, no. 5, pp. 250-258, May 2013.

[10] A. Ameri, E. J. Scheme, E. N. Kamavuako, K. B. Englehart, and P. A. Parker, "Real-time, simultaneous myoelectric control using force and position-based training paradigms," IEEE Trans. Biomed. Eng., vol. 61, no. 2, pp. 279-287, Feb. 2014.

[11] B. Hudgins, P. Parker, and R. N. Scott, "A new strategy for multifunction myoelectric control," IEEE Trans. Biomed. Eng., vol. 40, no. 1, pp. 82-94, Jan. 1993.

[12] J. L. G. Nielsen, S. Holmgaard, N. Jiang, K. B. Englehart, D. Farina, and P. A. Parker, "Simultaneous and proportional force estimation for multifunction myoelectric prostheses using mirrored bilateral training," IEEE Trans. Biomed. Eng., vol. 58, no. 3, pp. 681-688, Mar. 2011.

[13] S. Muceli and D. Farina, "Simultaneous and proportional estimation of hand kinematics from EMG during mirrored movements at multiple degrees-of-freedom.," IEEE Trans. Neural Syst. Rehabil. Eng., vol. 20, no. 3, pp. 371-378, May 2012.

[14] J. M. Hahne, H. Rehbaum, F. Biessmann, F. C. Meinecke, K.-R Muller, N. Jiang, D. Farina, and L. C. Parra, "Simultaneous and proportional control of 2D wrist movements with myoelectric signals," in Proc. IEEE Int. Work. Machine Learning Signal Processing, Sep. 2012, pp. 1-6.

[15] P. A. Parker, J. A. Stuller, and R. N. Scott, "Signal processing for the multistate myoelectric channel," Proc. IEEE, vol. 65, no. 5, pp. 662-674, May 1977.

[16] P. A. Parker and R. N. Scott, "Myoelectric control of prostheses," Crit. Rev. Biomed. Eng., vol. 13, no. 4, pp. 283-310, 1986.

[17] N. Jiang, P. A. Parker, and K. B. Englehart, "Spectrum of the nonstationary electromyographic signal modelled with integral pulse frequency modulation and its application to estimating neural drive information," J. Electromyogr. Kinesiol., vol. 19, no. 4, pp. e267-e279, Aug. 2009.

[18] L. Liu, P. Liu, E. a. Clancy, E. Scheme, and K. B. Englehart, "Electromyogram whitening for improved classification accuracy in upper limb prosthesis control," IEEE Trans. Neural Syst. Rehabil. Eng., vol. 21, no. 5, pp. 767-774, Sep. 2013.

[19] E. A. Clancy, E. L. Morin, and R. Merletti, "Sampling, noise-reduction and amplitude estimation issues in surface electromyography," $J$. Electromyogr. Kinesiol., vol. 12, no. 1, pp. 1-16, 2002.

[20] H. B. Evans, Z. Pan, P. A. Parker, and R. N. Scott, "Signal processing for proportional myoelectric control," IEEE Trans. Biomed. Eng., vol. 31, no. 2, pp. 207-211, Feb. 1984.

[21] T. D. Johnson, R. M. Elashoff, and S. J. Harkema, “A Bayesian changepoint analysis of electromyographic data: Detecting muscle activation patterns and associated applications," Biostatistics, vol. 4, no. 1, pp. 143-164, 2003.

[22] T. D. Sanger, "Bayesian filtering of myoelectric signals," J. Neurophysiol., vol. 97, no. 2, pp. 1839-1845, 2007.

[23] C. Gardiner, Stochastic Methods: A Handbook for the Natural and Social Sciences, 3rd ed. New York, NY, USA: Springer, 2009.

[24] Risken, The Fokker-Planck Equation, 2nd ed. Berlin, Germany: Springer, 1989.

[25] J. Kaipio, E. Somersalo, E. S. S. Antman, J. E. M. L. Sirovich, A. J. K. Hale, P. H. J. Keener, J. K. B. J. Matkowsky, and A. M. C. S. Peskin, Statistical Computational Inverse Problems, vol. 160, 2004. 
[26] E. Clancy and N. Hogan, "Probability density of the surface electromyogram and its relation to amplitude detectors," IEEE Trans. Biomed. Eng., vol. 46, no. 6, pp. 730-739, Jun. 1999.

[27] N. Jiang, I. Vujaklija, H. Rehbaum, B. Graimann, and D. Farina, "Is accurate mapping of EMG signals on kinematics needed for precise online myoelectric control?," IEEE Trans. Neural Syst. Rehabil. Eng., vol. 22, no. 3, pp. 549-558, May 2014.

[28] S. Muceli, N. Jiang, and D. Farina, "Extracting signals robust to electrode number and shift for online simultaneous and proportional myoelectric control by factorization algorithms.," IEEE Trans. Neural Syst. Rehabil. Eng., vol. 22, no. 3, pp. 623-633, May 2014.

[29] Google, Inc., Android Metrics and Grids [Online]. Available: http:// developer.android.com/design/style/metrics-grids.html

[30] M. R. Williams and R. F. Kirsch, "Evaluation of head orientation and neck muscle EMG signals as command inputs to a human-computer interface for individuals with high tetraplegia," IEEE Trans. Neural Syst. Rehabil. Eng., vol. 16, no. 5, pp. 485-496, Oct. 2008.

[31] R. Bloom, A. Przekop, and T. D. Sanger, "Prolonged electromyogram biofeedback improves upper extremity function in children with cerebral palsy," J. Child Neurol., vol. 25, no. 12, pp. 1480-1484, 2010.

[32] D. Graupe and W. K. Cline, "Functional separation of EMG signals via ARMA identification methods for prosthesis control purposes," IEEE Trans. Systems, Man Cybern., vol. SMC-5, no. 2, pp. 252-259, Apr. 1975.

[33] K. Nazarpour, C. Ethier, L. Paninski, J. M. Rebesco, R. C. Miall, and L. E. Miller, "EMG prediction from motor cortical recordings via a nonnegative point-process filter," IEEE Trans. Biomed. Eng., vol. 59, no. 7, pp. 1829-1838, Jul. 2012.

[34] P. Kumar, C. Potluri, A. Sebastian, S. Chiu, A. Urfer, D. S. Naidu, and M. P. Schoen, "An adaptive multi sensor data fusion with hybrid nonlinear ARX and Wiener-Hammerstein models for skeletal muscle force estimation," in Proc. 14th WSEAS Int. Conf. Syst., 2010, vol. I, no. Vol. I, pp. 186-191.

[35] M. Anugolu, A. Sebastian, P. Kumar, M. P. Schoen, A. Urfer, and D. S. Naidu, "Surface EMG array sensor based model fusion using Bayesian approaches for prosthetic hands," in Proc. ASME Dynamic Systems Control Conf., 2009, pp. 721-723.

[36] T. Pistohl, C. Cipriani, A. Jackson, and K. Nazarpour, "Abstract and proportional myoelectric control for multi-fingered hand prostheses," Ann. Biomed. Eng., vol. 41, no. 12, pp. 2687-2698, 2013.

[37] P. Krishnaswamy, E. N. Brown, and H. M. Herr, "Human leg model predicts ankle muscle-tendon morphology, state, roles and energetics in walking," PLoS Comput. Biol., vol. 7, no. 3, 2011.

[38] S. J. Young, J. van Doornik, and T. D. Sanger, "Finger muscle control in children with dystonia," Mov. Disord., vol. 26, no. 7, pp. 1290-1296, 2011.

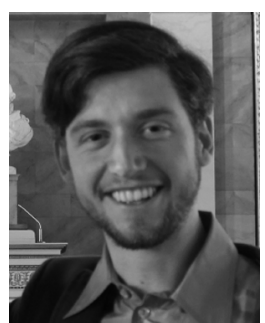

David Hofmann received the Dipl.-Phys. degree in physics from the Technische Universität München, Munich, Germany, in 2008, and the Ph.D. degree in physics.

$\mathrm{He}$ is currently employed as a Postdoctoral Researcher at the Department for Theoretical Physics, Emory University, Atlanta, GA, USA. His research interests include biosignal analysis, biophysical modeling of single cell electrophysiology, neural control of movement, Bayesian modeling of decision making.

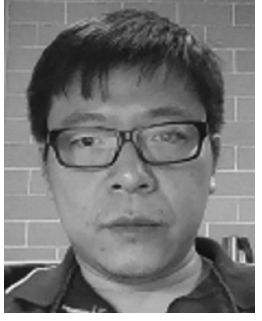

Ning Jiang ( $\left.\mathrm{S}^{\prime} 02-\mathrm{M}^{\prime} 09\right)$ received the B.S. degree in electrical engineering from Xi'an Jiaotong University, Xi' an, China, in 1998, and the M.Sc. and Ph.D. degrees in engineering from the University of New Brunswick, Fredericton, NB, Canada, in 2004 and 2009, respectively.

He was Research Assistant Professor at Aalborg University, Denmark, from 2009 to 2010, a Marie Curie Fellow at the Strategic Technology Management, Otto Bock Healthcare GmbH, Germany, from 2010 to 2012, and a Research Scientist with the Department of Neurorehabilitation Engineering, University Medical Center Göttingen, Georg-August University, Göttingen, Germany, from 2012 to 2015. He is currently an Assistant Professor at the Department of Systems Design Engineering, University of Waterloo, Waterloo, Canada. His research interests include signal processing of electromyography, advanced prosthetic control, neuromuscular modeling, and BCI for neurorehabilitation.

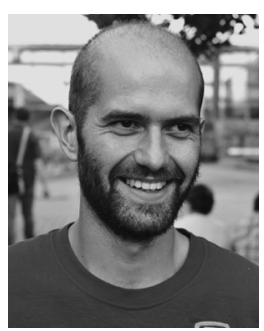

Ivan Vujaklija (S'14) received the Dipl.-Ing. degree in electrical engineering and computer science from the University of Belgrade, Serbia, in 2011, and the M.Sc. degree in biomedical engineering from the University of Lübeck, Germany, in 2013. He is working toward the Ph.D. degree in human medical sciences at the University of Göttingen, Germany.

Currently, he works as a Research Assistant at the Institute for Neurorehabilitation Systems at the University Medical Center Göttingen, Georg-August University, Germany. His research interests include biosignal processing, advance control algorithms, robotics, neurorehabilitation and neural control of movement.

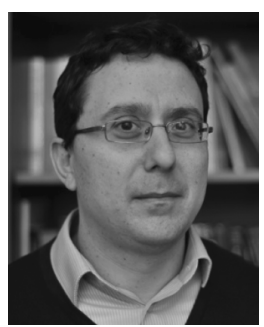

Dario Farina (SM'13) received the Ph.D. degree in automatic control and computer science from the Ecole Centrale de Nantes, Nantes, France, in 2001, and the Ph.D. degree in electronics and communications engineering from Politecnico di Torino, Italy, in 2002 .

After working as a Research Scientist at the Laboratory for Neuromuscular System Engineering (LISiN) at Politecnico di Torino, he was an Associate Professor in Biomedical Engineering at Aalborg University, Aalborg, Denmark, from 2004 to 2008. At the same University, in 2008, he became a Full Professor in motor control and biomedical signal processing and the Head of the Research Group on Neural Engineering and Neurophysiology of Movement. In 2010, he was appointed Full Professor and Founding Chair of the Department of Neurorehabilitation Engineering at the University Medical Center Göttingen, Georg-August University, Germany, within the Bernstein Focus Neurotechnology (BFNT) Göttingen. In this position, he has also been the Chair for NeuroInformatics of the BFNT Göttingen since 2010. His research focuses on neurorehabilitation technology, neural control of movement, and biomedical signal processing and modelling. Within these areas, Prof. Farina has (co)-authored more than 350 papers in peer-reviewed journals and over 400 among conference papers/abstracts, book chapters, and encyclopedia contributions. $\mathrm{He}$ has been the (co-)Editor of the IEEE/Wiley books Introduction to Neural Engineering for Motor Rehabilitation (2013) and Surface Electromyography: Physiology, Engineering and Applications (2015).

Prof. Farina was the President of the International Society of Electrophysiology and Kinesiology (ISEK) from 2012 to 2014. Among other awards, he has been the recipient of the 2010 IEEE Engineering in Medicine and Biology Society Early Career Achievement Award. He is an Associate Editor of IEEE TRANSACTIONS ON BIOMEDICAL ENGINEERING, Journal of Physiology, Medical \& Biological Engineering \& Computing, and the Journal of Electromyography and Kinesiology. 